\title{
Fungal and mold hazards in forensic science laboratories
}

\author{
Vinod Dhingra ${ }^{1, *}$, Jyotsna Pandey ${ }^{2}$ \\ Senior Scientific Officer, Forensic Sciences Laboratory, Sagar, Madhya Pradesh, India
}

*Corresponding Author:

Email: vdhingraso@hotmail.com

\begin{abstract}
Forensic science aims to serve society by advancing justice. It is important that forensic scientists should aware of mold and fungal growth in their laboratory premises and should take actions, wherever possible, to reduce the growth of harmful fungi as well as unnecessary potential hazard. The mycotoxins produced by these fungi create several diseases. The present scenario of mold and fungi management in Indian forensic laboratories is grim. However there is an emerging concern regarding fungi management. It should be mandatory for the forensic laboratories to ensure any adverse effect to human health and environment. This study intends to create awareness regarding identification, prevention and remediation of fungi, mold and mycotoxins in forensic laboratories.
\end{abstract}

Keywords: Fungi, Mold, Mycotoxins, Forensic laboratories.

\section{Introduction}

Mold and fungi is considered a subset of indoor air quality, their thousands of species remains present in virtually in every environment and can be detected, indoors and outdoors, round the year. Molds and fungi produce and release millions of spores, small enough to be air, water or insect-borne. They can also produce toxic self-defense agents known as mycotoxins, which produce negative effects on human health including allergic reactions, asthma and other respiratory problems. Indoor moisture leading to the growth of molds may come from flooding, water intrusion, high humidity, condensation and steam. Molds need moisture and organic material as a food source for their growth. Molds can be of any color, including white, orange, green, brown, or black and produces mildew or earthy smell. They generally have seen in indoors on wet/damp walls, carpets, ceilings, or behind wallpaper and laminates as well as in heating, ventilating, and air conditioners systems. This study intends to create awareness regarding identification, prevention and remediation of fungi, mold and mycotoxins in forensic laboratories. ${ }^{1,5}$
Growth of Mold and Fungi in Forensic Laboratories: Forensic laboratories conduct examination of exhibits of various criminal cases including autopsy material, pesticides, food materials, vomit's material, cloths of deceased persons, remnants of dead body, skeletons, poisonous material, biological fluids like blood, semen, saliva, hair, stool etc. This contains protein material and attracts various microorganisms like fungi and mold which deteriorates the atmosphere of forensic laboratories and affect the health of forensic experts and associated staff.

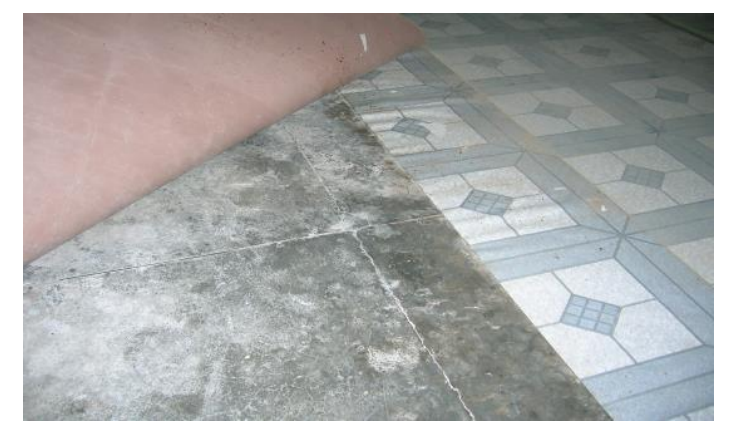

Fig. 1: Mold and fungal spores below the vinyl floorings

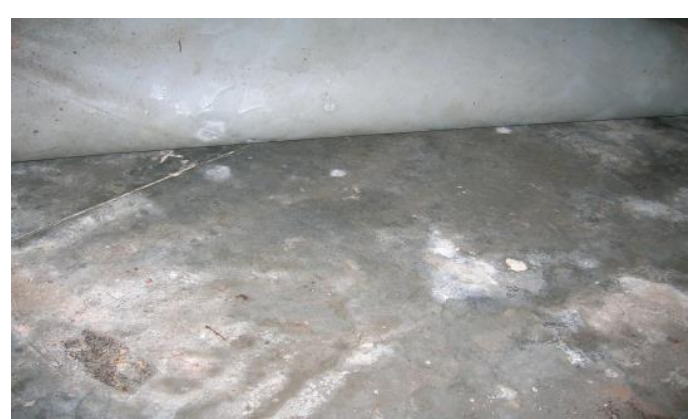

Fig. 2: Growth of mold and fungal spores below the carpet

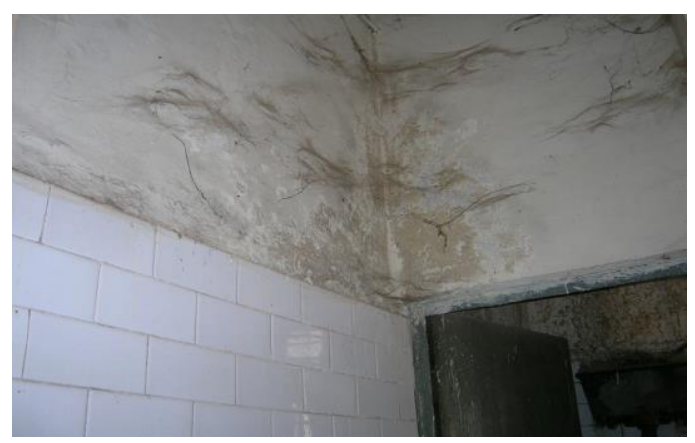

Fig. 3: Fungal spores ideally grown at unattended toilets side needs attention 


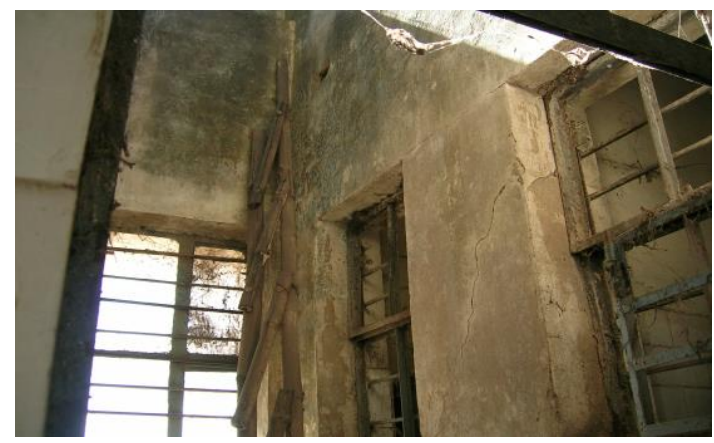

Fig. 4: Fungal spores at unattended damp areas

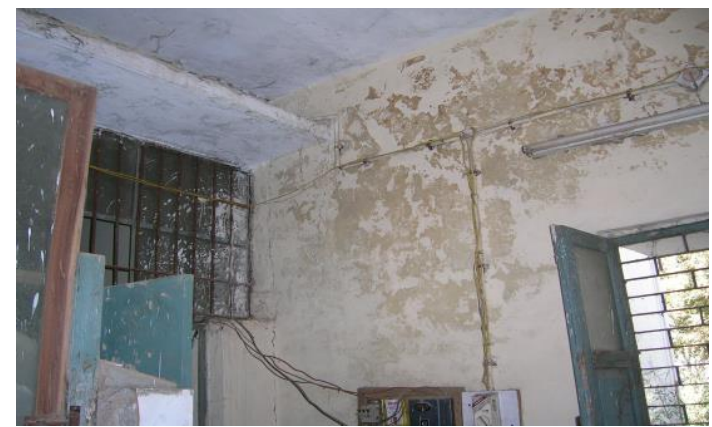

Fig. 5: Ideal space for growth of fungal spores

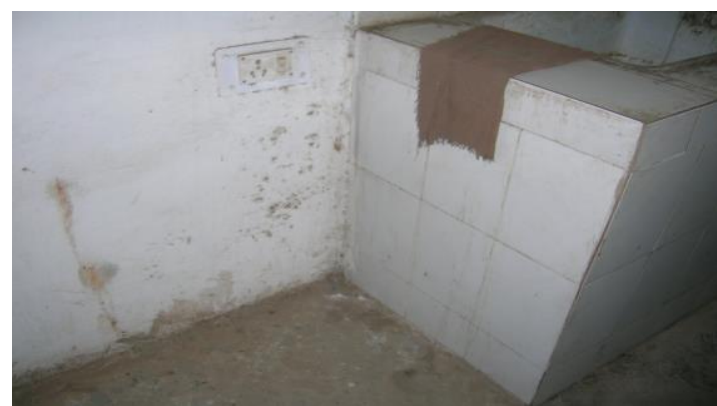

Fig. 6: Below the sink moist area showing spores of fungi and mold

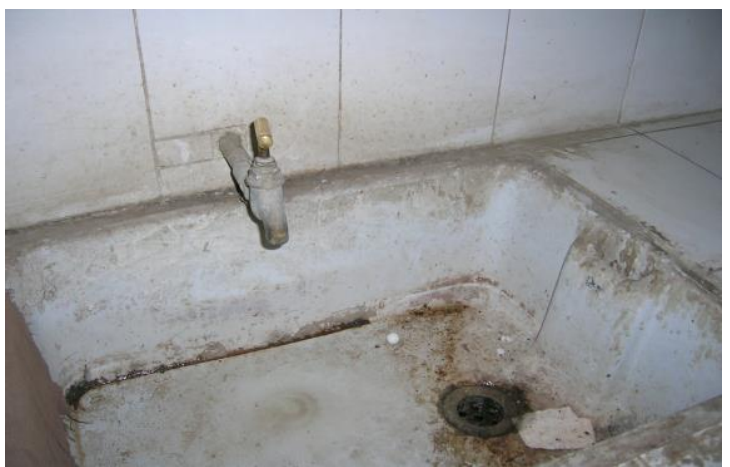

Fig. 7: Unattended biological fluid material when left in wash basin creates ideal medium for growth of fungi and mold

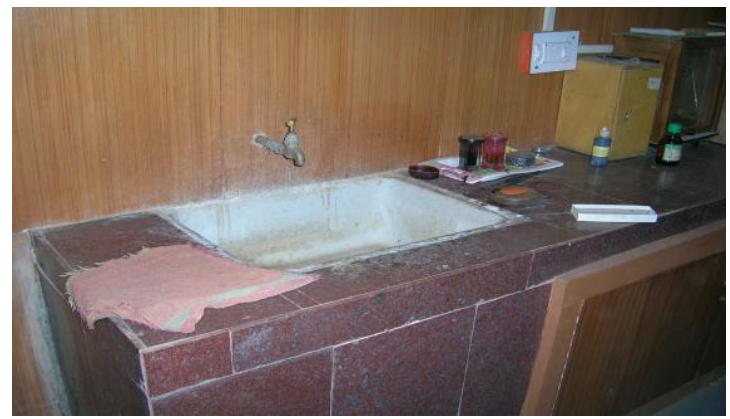

Fig. 8: Wooden material contains biological material provides place for fungi and molds.

Relocation, Prevention and Health Effects on forensic personnel's: Persons diagnosed with fungal related diseases should avoid work to the affected areas until remediation and air testing are complete, except in cases of wide spread fungal contamination that are linked to illnesses. In order to prevent or reduce mold growth and exposure training of staff like prevention of moisture, discarding of fungi and mold affected articles, routine inspection of building is necessary.

Fungi, molds and mycotoxins can cause allergic reactions, fungal infections, and other health effects. Inhalation of fungal spores, fragments, or metabolites from a wide variety of fungi may lead to toxic effects, or cause infections in some professional. The most common symptoms reported from exposures in indoor environments are runny nose, eye irritation, and cough, and congestion, aggravation of asthma, headache, and fatigue during skin contact and respiration. The symptoms during exposer to fungi depends on the nature of the fungal material, the amount of exposure, and the susceptibility of exposed persons which varies with the genetic predisposition, age, state of health, and concurrent exposures. Hypersensitivity pneumonitis may occur after repeated exposures to an allergen and can result in permanent lung damage to the affected person in some cases. Several species of Aspergillus are known to cause aspergillosis.

Detection and Laboratory Analysis: Fungi and mold can be detected by visual inspection which is the most important initial step in identifying a possible contamination problem. The extent of any water damage and mold growth should be visually assessed, it is important in determining remedial strategies. Ventilation systems should also be visually checked, particularly for damp filters and damp conditions elsewhere in the system and overall cleanliness. The use of instrument and equipment such as a bore scope, to view spaces in ductwork or behind walls, or a moisture meter, to detect moisture in building materials, may be helpful in identifying hidden sources of fungal growth and the extent of water damage. Mold can also be detected by tape lift, Air-O-Cell filter. Bulk samples are usually collected from visibly moldy surfaces by scraping or cutting materials with a clean tool into a clean plastic bag. Surface samples are usually collected 
by wiping a measured area with a sterile swab or by stripping the suspect surface with clear tape. A mycologist should be consulted for specific sampling and delivery instructions and chain-of-custody forms.

Microscopic identification of the spores/colonies requires considerable expertise. These services are not routinely available from commercial laboratories. QPCR and Polymerase chain, reaction are now day's available methods for detection of fungi and molds.

\section{Remediation}

The moisture must be eliminated otherwise mold will return. The goal of remediation is to remove or clean contaminated materials in a way that prevents the emission of fungi and dust contaminated with fungi from entrance and exit of work area protecting the health of workers performing the abatement.

The exposure to mold often occurs during remediation process. Depending on remediation tasks which depends on the size of the area for small isolated areas e.g., ceiling tiles, small areas on walls. Remediation can be conducted by regular building maintenance staff using proper protection to avoid health hazards. For middle and big sized area plastic sheets, tapes and vaccume equipped with high efficiency particulate air filters can be used, larger areas like ventillation, grills, heavy air conditioners etc. remediation needs professionals. During remediation process respiratory protection, gloves and eye protection is recommended and contaminated materials that cannot be cleaned should be removed from the building in a sealed plastic bag. The work area and areas used by remedial workers for egress should be cleaned with a damp cloth and/or mop and a detergent solution. All areas should be left dry and visibly free from contamination and debris.

\section{Conclusion}

He infestation of fungi, molds and mycotoxins in forensic laboratories, in addition to the posing risk to the scientist and personnel who works in laboratories, is also a threat to the public health and environment. It is emerging as a health hazard to the forensic personnel's at large. Keeping in view, checking the problem fungi, molds and mycotoxins the management should take effective steps to protect the forensic personnel's and society. The most imperative component of the fungi and mold management plans is to develop a system and culture through education, training and persistent motivation of the forensic services staff.

\section{Acknowledgements}

We are thankful to Director, Dr. Harsh Sharma, Director, Forensic Science Laboratory, Sagar and Rt. Joint director, Mr. S. R. Patidar, Regional Forensic Science Laboratory Gwalior M. P. for valuable support and motivation.

\section{References}

1. Barrett, J. 2000. Mycotoxins: of molds and maladies. Environ. Health Perspect. 108:A20-A23.

2. Hayes, A. W. 1980. Mycotoxins: a review of biological effects and their role in human diseases. Clin Toxicol. 17:45-83.

3. Cole, R. J., and R. H. Cox. 1981. Handbook of toxic fungal metabolites. Academic Press, New York, N.Y.

4. Sternberg, S. 1994. The emerging fungal threat. Science 266:1632-1634.

5. Bhatnagar, D., E. B. Lillehoj, and D. K. Arora. 1992. Handbook of applied mycology, vol. 5. Mycotoxins in ecological systems. Marcel Dekker, Inc., New York, N.Y. 